\title{
A study of the energy spectrum and composition of cosmic rays up to $10^{18} \mathrm{eV}$
}

\author{
S. Thoudam ${ }^{* 1}$, A. Achterberg ${ }^{1}$, S. Buitink ${ }^{2}$, H. Falcke ${ }^{1,3,4}$, J.R. Hörandel ${ }^{1,3}$, \\ J.P. Rachen ${ }^{1}$ \\ 1 Department of Astrophysics/IMAPP, Radboud University Nijmegen, P.O. Box 9010, 6500 GL \\ Nijmegen, The Netherlands \\ 2 Astrophysical Institute, Vrije Universiteit Brussel, Pleinlaan 2, 1050 Brussels, Belgium \\ 3 NIKHEF, Science Park Amsterdam, 1098 XG Amsterdam, The Netherlands \\ 4 Netherlands Institute of Radio Astronomy (ASTRON), Postbus 2, 7990 AA Dwingeloo, The \\ Netherlands \\ E-mail: s.thoudameastro.ru.nl
}

\begin{abstract}
Motivated by the recent high-precision measurements of the cosmic-ray energy spectrum and composition by several new-generation experiments, we have conducted a detailed study to understand the observed properties of cosmic rays up to $\sim 10^{18} \mathrm{eV}$. The study involves building a propagation model for cosmic rays originating from supernova explosions in the interstellar medium. Although these cosmic rays can satisfactorily explain the observed spectra of different elements at low energies provided by balloon and satellite borne experiments, we found that they cannot account for the cosmic rays above $\sim 10^{16} \mathrm{eV}$ observed by air shower experiments. An additional component of Galactic cosmic rays is required in order to explain the observed cosmic rays beyond this energy up to $\sim 10^{18} \mathrm{eV}$. Possible scenarios for this additional component, and comparison with the observed all-particle spectrum and composition are presented.
\end{abstract}

The 34th International Cosmic Ray Conference,

30 July- 6 August, 2015

The Hague, The Netherlands

\footnotetext{
* Speaker.
} 


\section{Introduction}

New-generation experiments have provided high-precision measurements of cosmic rays up to energies exceeding $10^{20} \mathrm{eV}$. At low energies, below $\sim 10^{15} \mathrm{eV}$, balloon and satellite borne experiments such as ATIC [24], CREAM [33], TRACER [23], PAMELA [8] and AMS [9] have measured the energy spectra of various species of both the hadronic and leptonic components of cosmic-rays. New results from these experiments, such as the rise in the positron fraction above $\sim 10 \mathrm{GeV}$ and the spectral hardening of proton and helium nuclei at $\mathrm{TeV}$ energies, are found to be at odds with our general understandings about the origin of cosmic rays and the nature of their propagation in the Galaxy. At high energies, above $\sim 10^{15} \mathrm{eV}$, air shower experiments like KASCADE-Grande [12], Tibet III array [10], IceTop [2], Pierre Auger Observatory [6] and Telescope Array [5] have provided detailed measurements of the all-particle energy spectrum and the composition of cosmic rays. In this energy regime, the origin of spectral features in the all-particle cosmic-ray spectrum such as the "knee" at $\sim 3 \times 10^{15} \mathrm{eV}$, the "second knee" at $\sim 3 \times 10^{17} \mathrm{eV}$, and the "ankle" at $\sim 5 \times 10^{18} \mathrm{eV}$, still remains unsettled, while the observed steepening at $\sim 4 \times 10^{19} \mathrm{eV}$ is commonly interpreted as the "GZK cut-off". Moreover, the behaviour of the observed composition in this energy further complicates the issue. The aim of this work is to build a model that explains all the observed features in the energy spectrum and the composition of cosmic rays up to $\sim 10^{18} \mathrm{eV}$.

\section{Cosmic rays from supernova explosions in the interstellar medium (SNR-CRs)}

It is generally assumed that supernova remnants present in the interstellar medium are the main sources for cosmic rays of energies up to around the knee [18]. This view is supported both by our theoretical understandings and observational evidences of supernova remnants in the Galaxy. Diffusive shock acceleration theory predicts that strong shock waves inside supernova remnants can accelerate particles with a power-law spectrum of index close to 2 up to energies close to the knee [13]. At the same time, the detection of non-thermal $\mathrm{X}$-rays and $\mathrm{TeV}$ gamma rays from a number of supernova remnants provide direct evidence for the presence of high-energy particles inside supernova remnants $[25,7]$. In particular, the detection of $\mathrm{TeV}$ gamma rays from the supernova remnant RX J1713.7-3946 with energies up to $\sim 100 \mathrm{TeV}$ indicates that high energy particles with energies up to $\sim 1 \mathrm{PeV}$ can be produced inside supernova remnants [7]. Once released from the sources, cosmic rays undergo diffusive propagation in the Galaxy due to the scattering by magnetic field irregularities present in the interstellar medium. During the propagation, it is possible that cosmic rays again interact with expanding supernova shock waves, and get re-accelerated. This re-acceleration process is expected to occur mainly with old supernova remnants since they occupy a larger volume in the Galaxy than the younger remnants. This model of cosmic-ray propagation, described in detail in Ref. [31], can be mathematically represented by the equation,

$$
\nabla \cdot(D \nabla N)-[\bar{n} v \sigma+\xi] \delta(z) N+\left[\xi_{s p^{-s}} \exp \left(-\frac{A p}{Z p_{\mathrm{r}}}\right) \int_{p_{0}}^{p} d u N(u) u^{s-1}\right] \delta(z)=-Q \delta(z),
$$

where a cylindrical geometry has been adopted for the propagation region described by the the radial and vertical coordinates $(r, z)$. The region is assumed to be bounded at $z= \pm L$, and no boundary in the $r$ direction. $N(r, z, p)$ represents the number density of cosmic-ray nuclei with 


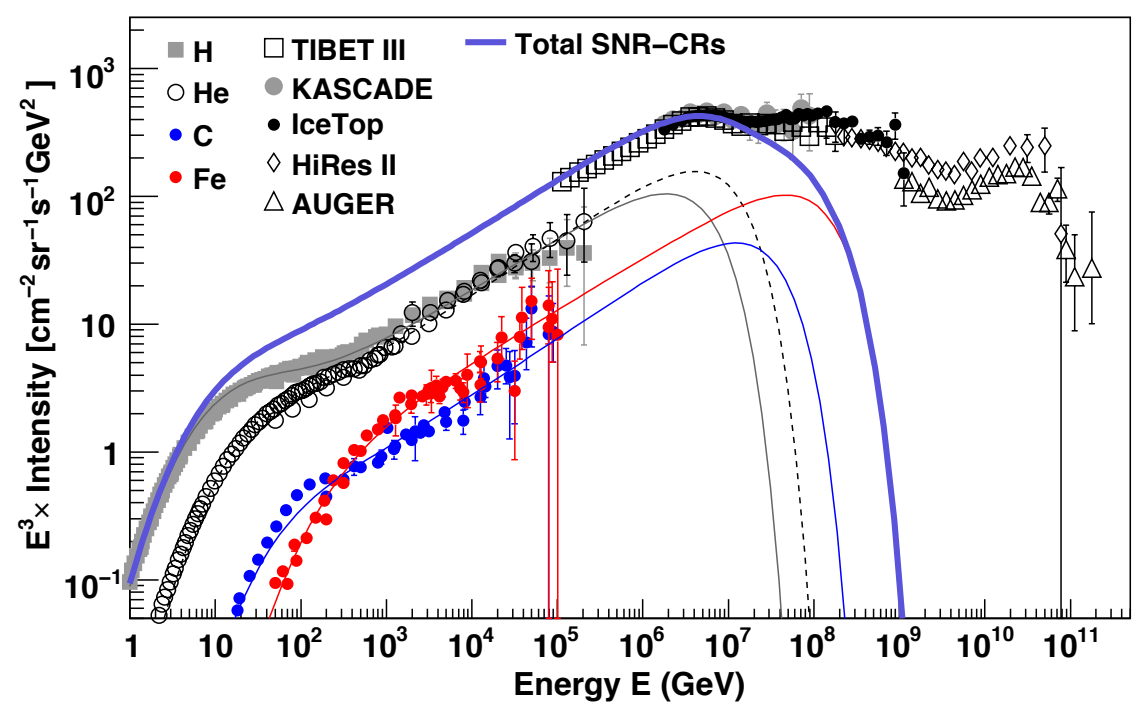

Figure 1: Contribution of SNR-CRs to the all-particle cosmic-ray spectrum. Thin lines: Spectra for protons, helium, carbon and oxygen nuclei, and Thick-solid line: Total contribution. Other nuclei (oxygen, neon, magnesium and silicon) are also included in the calculation, but are not shown here. Low energy data: See references given in [31]. High energy data: KASCADE [11], IceTop [2], Tibet III [10], AUGER [6], and HiRes II [3]. See text for the values of model parameters.

momentum per nucleon $p, D(\rho)=D_{0}\left(\rho / \rho_{0}\right)^{a}$ is the diffusion coefficient as a function of the particle rigidity $\rho$, where $\left(D_{0}, \rho_{0}, a\right)$ are constants, $\bar{n}$ is the surface matter density in the Galactic disk, $\sigma(p)$ the inelastic interaction cross-section, $\xi$ corresponds to the rate of re-acceleration, and $Q(r, p) \delta(z)$ is the source term. The re-acceleration, represented by the term containing the integral in Equation 2.1, is assumed to produce a power-law spectrum of particles with an index $s$ and an exponential cut-off in the total momentum at $Z p_{\mathrm{r}}$, where $Z$ denotes the charge number of the nuclei. The sources are assumed to be distributed in the Galactic disk and extended up to a radius $R_{\mathrm{g}}$. We write $Q(r, p)=\bar{v} H\left[R_{\mathrm{g}}-r\right] H\left[p-p_{0}\right] Q(p)$, where $\bar{v}$ is the frequency of supernova explosions per unit surface area in the Galactic disk, $H(m)=1(0)$ for $m>0(<0)$ represents a Heaviside step function, and the source spectrum $Q(p)$ as,

$$
Q(p)=A Q_{0}(A p)^{-q} \exp \left(-\frac{A p}{Z p_{\mathrm{c}}}\right)
$$

where $Q_{0}$ is constant proportional to the amount of supernova kinetic energy injected into cosmic rays, $A$ is the mass number of the cosmic rays, $q$ is the source spectral index, and $p_{\mathrm{c}}$ is the cutoff momentum for protons. We take $\xi=\eta V \bar{v}$, where $V=4 \pi R_{\mathrm{sn}}^{3} / 3$ is the volume of a supernova remnant re-accelerating the cosmic rays with $R_{\mathrm{sn}}$ representing its radius, and $\eta$ is a correction factor that is introduced to account for the actual unknown size of the remnants.

From the analytical solution of Equation 2.1 [31], the cosmic-ray densities for different elements are obtained, and compared with the measurements at low energies. For the calculation, we take $L=5 \mathrm{kpc}, R_{\mathrm{g}}=20 \mathrm{kpc}, R_{\mathrm{sn}}=100 \mathrm{pc}, \bar{n}=7.24 \times 10^{20}$ atoms $\mathrm{cm}^{-2}$ with an additional $10 \%$ helium atoms, and $\bar{v}=25 \mathrm{SNe} \mathrm{Myr}^{-1} \mathrm{kpc}^{-2}$ which corresponds to a supernova explosion frequency of $\sim 1 / 30 \mathrm{yr}^{-1}$ [30]. The cosmic-ray propagation $\left(D_{0}, \rho_{0}, a\right)$ and the re-acceleration parameters 
$(\eta, s)$ are taken as, $D_{0}=9 \times 10^{28} \mathrm{~cm}^{2} \mathrm{~s}^{-1}, \rho=3 \mathrm{GV}, a=0.33, \eta=1.02$, and $s=4.5$ [31]. Also, the predicted all-particle spectrum is compared with the measured all-particle spectrum at high energies. We found that taking an exponential energy cut-off for protons at $E_{\mathrm{c}}=4.5 \times 10^{6} \mathrm{GeV}$ reproduces the observed knee. The results are shown in Figure 1. For clarity, the individual spectra of only the protons, helium, carbon and iron nuclei are shown. Other nuclei which are sub-dominant, i.e., oxygen, neon, magnesium and silicon nuclei, are also included in the calculation, but they are not shown in the figure. It can be noticed that SNR-CRs cannot account for the observed cosmic rays above $\sim 10^{7} \mathrm{GeV}$. They contribute only $\sim 30 \%$ at $\sim 10^{8} \mathrm{GeV}$. As extra-galactic cosmic rays presumably dominates only above $\sim 10^{9} \mathrm{GeV}$, our result suggests that an additional component of Galactic cosmic rays is required in order to explain the observed spectrum above $\sim 10^{7} \mathrm{GeV}$ (see also [19]).

\section{Additional component of Galactic cosmic rays}

Here, we consider two possible origins for an additional component. One is the re-acceleration of SNR-CRs by the Galactic wind termination shocks (Wind-CRs), and the other is the contribution of cosmic rays from Wolf-Rayet star supernova explosions in the Galaxy (WR-CRs).

\subsection{Cosmic rays from Galactic wind termination shocks (Wind-CRs)}

Presuming that Galactic wind exists (see e.g., [22]), at distance far away from the Galactic disk, roughly at around $100 \mathrm{kpc}$ or even more, the wind flow is expected to terminate resulting into the formation of termination shocks [34]. These shocks can re-accelerate the SNR-CRs escaping into the Galactic halo to higher energies [20,35]. The propagation of escaped SNR-CRs in the Galactic wind region can be described by,

$$
\nabla .\left(D_{\mathrm{w}} \nabla N_{\mathrm{w}}-\mathbf{V} N_{\mathrm{w}}\right)+\frac{\partial}{\partial p}\left\{\frac{\nabla \cdot \mathbf{V}}{3} p N_{\mathrm{w}}\right\}=-Q_{\mathrm{esc}} \boldsymbol{\delta}(\mathbf{r}),
$$

where we assume a spherically symmetric geometry with radius coordinate $r, D_{\mathrm{w}}$ is the diffusion coefficient in the wind region, $N_{\mathrm{w}}(r, p)$ is the number density of cosmic rays, $\mathbf{V}=V_{0} \mathbf{r}$ is the wind velocity which is assumed to be directed radially outwards, and $Q_{\text {esc }}$ is the total escape rate of SNRCRs from the diffusion boundary (at $z= \pm L$ ). Since the size of the escaping region is expected to be much less than the size of the termination shock, we assume that $Q_{\text {esc }}$ is produced by a point source located at $r=0$. Using the solution of Equation 3.1 [32], we calculate the total rate of cosmic rays $Q_{\mathrm{inj}}(p)$ injected into the termination shock, which is taken to be located at a distance $r=R_{\mathrm{sh}}$. Assuming that only a certain fraction $k_{\mathrm{sh}}$ participates in the re-acceleration process, the cosmic-ray spectrum produced by the termination shock can be written as [17],

$$
Q_{\mathrm{sh}}(p)=\gamma p^{-\gamma} \exp \left(-\frac{A p}{Z p_{\mathrm{sh}}}\right) \int_{p_{0}}^{p} k_{\mathrm{sh}} Q_{\mathrm{inj}}(u) u^{\gamma-1} d u
$$

where $\gamma$ is the spectral index, and we have introduced an exponential cut-off in the spectrum at $Z p_{\text {sh }}$ with $p_{\text {sh }}$ representing the cut-off momentum for protons.

After re-acceleration, some of the re-accelerated cosmic rays can return to the Galactic disk through diffusive propagation against the Galactic wind flow. The propagation can be described 
also by Equation 3.1. Neglecting adiabatic losses, the density of re-accelerated cosmic rays reaching the Earth is given by [32],

$$
N_{\text {Wind }-\mathrm{CRs}}(p)=\frac{Q_{\mathrm{sh}}}{4 \pi D_{\mathrm{w}} R_{\mathrm{sh}}} \exp \left[-\frac{V_{0} R_{\mathrm{sh}}^{2}}{2 D_{\mathrm{w}}}\right] .
$$

The diffusion in the wind region is assumed to be much faster than near the Galactic disk, but has the same rigidity dependence as $D$. We take $D_{\mathrm{w}}=10 \mathrm{D}$. For the wind velocity, we take $V_{0}=15 \mathrm{~km} / \mathrm{s} / \mathrm{kpc}$ [16]. The distance to the termination shock is determined based on the condition that the Galactic wind ram pressure is equal to the intergalactic pressure at the position of the shock. For an intergalactic pressure of $10^{-15} \mathrm{ergs} \mathrm{cm}^{-3}$ [35], and assuming a Galactic wind driven by SNR-CRs [34], we obtain $R_{\mathrm{sh}}=96.2 \mathrm{kpc}$.

\subsection{Cosmic rays from Wolf-Rayet star supernova explosions (WR-CRs)}

Although most of supernova explosions in the Galaxy occur in the interstellar medium, a small fraction is expected to occur into the winds of massive stars like Wolf-Rayet stars. Magnetic fields in the wind of Wolf-Rayet stars can reach more than 100 Gauss, and a strong supernova shock in such a field can accelerate particles up to energies close to $10^{9} \mathrm{GeV}[15,29]$. We assume that Wolf-Rayet stars are also distributed in the Galactic disk, and the propagation of cosmic rays from these stars (WR-CRs) is described by Equation 2.1. The source spectrum is also described by Equation 2.2. We assume a Wolf-Rayet supernova explosion frequency of $1 / 210 \mathrm{yr}^{-1}$ in the Galaxy [28]. This corresponds to roughly 1 Wolf-Rayet explosion for every 7 supernova explosions in the Galaxy. The source abundance ratios of the different elements is assumed to be proportional to the relative abundances of elements in the wind. We use the relative abundances with respect to helium for a specific case of carbon-to-helium $(\mathrm{C} / \mathrm{He})$ ratio of 0.4 given in Ref. [26]. Only the overall normalisation of the total WR-CRs and the maximum energy of the proton source spectrum are taken as model parameters. They are optimised based on the observed all-particle spectrum between $\sim 10^{8}$ and $10^{9} \mathrm{GeV}$.

\section{All-particle spectrum and the composition at high energies}

In Figure 2 (top panel), we show the all-particle spectrum obtained by combining the contributions of the SNR-CRs and the Wind-CRs along with the measured data. Also included in the total spectrum is the contribution of the extra-galactic cosmic rays taken from Ref. [27], modified for energy above $4 \times 10^{10} \mathrm{GeV}$ to fit the measured data, which represents a pure proton population for an input source spectrum of $E^{-2}$. For the SNR-CRs, the maximum cut-off energy is reduced to $E_{\mathrm{c}}=3.3 \times 10^{6} \mathrm{GeV}$ from the value of $4.5 \times 10^{6} \mathrm{GeV}$ used in Figure 1 in order to better reproduce the measurements around the knee. For the Wind-CRs, we take a particle injection fraction of $k_{\mathrm{sh}}=18.5 \%$ into the re-acceleration process, and an exponential cut-off energy for protons (corresponding to $p_{\mathrm{sh}}$ in Equation 3.2) of $E_{\mathrm{sh}}=1.3 \times 10^{8} \mathrm{GeV}$. The model nicely reproduces the observed knee, but it does not predict a prominent second knee as observed. At the same time, the ankle predicted by the model lies at somewhat higher energy than the observed ankle position. However, overall, the predicted total spectrum still seems to agree with the observed spectrum within the systematic uncertainties of the different measurements. 

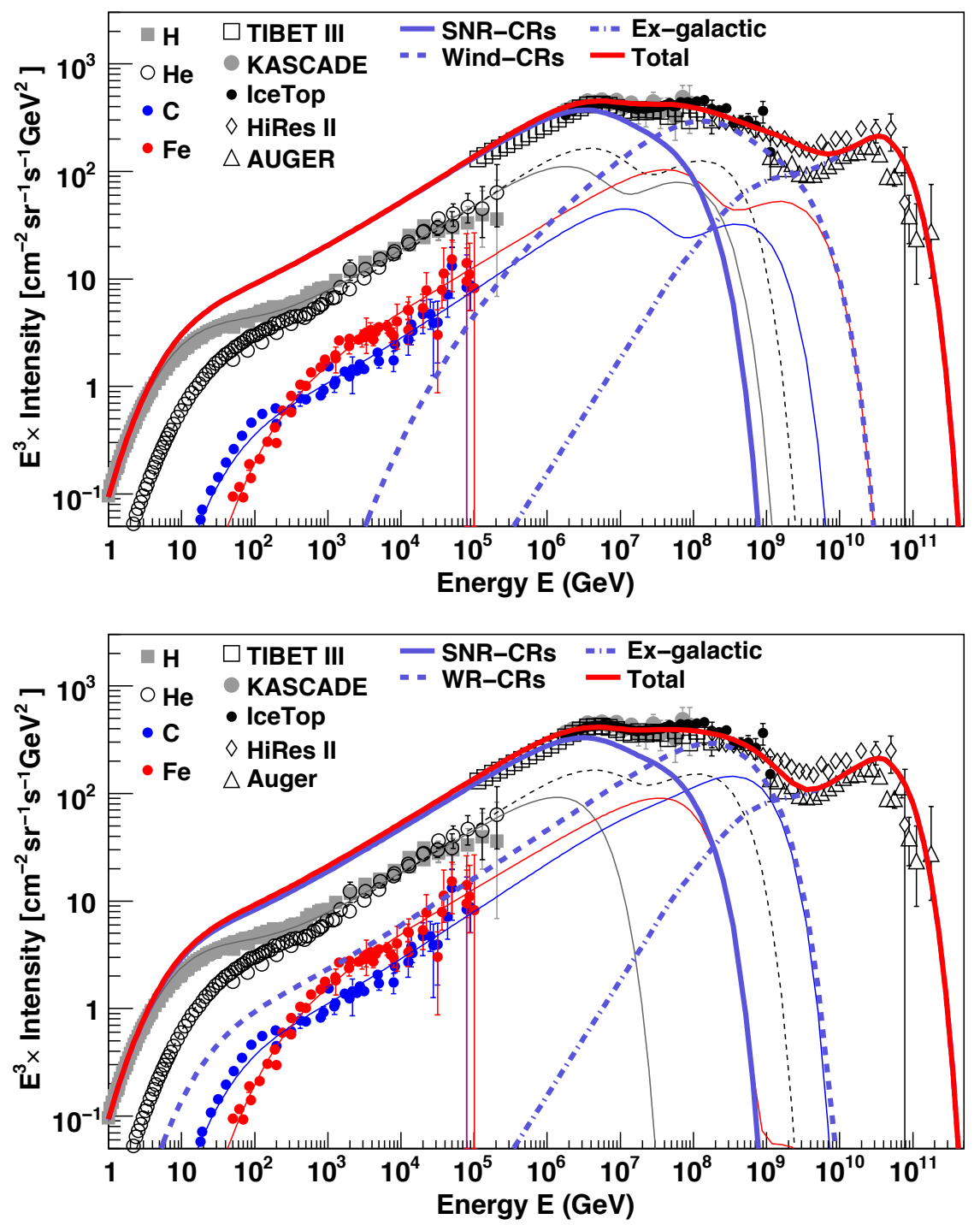

Figure 2: Model prediction for the all-particle energy spectrum using the Galactic wind re-acceleration (top) and the Wolf-Rayet stars (bottom) models. Thick-solid blue line: Total SNR-CRs, Thick-dashed line: Wind-CRs (or WR-CRs), Thick-dot-dashed line: Extra-galactic component, Thick-solid red line: Total allparticle spectrum, and Thin lines: SNR-CRs plus Wind-CRs (or WR-CRs) for protons, helium, carbon and iron nuclei. Other nuclei (oxygen, neon, magnesium and silicon) are also included in the calculation, but are not shown in the figures. See text for the values of model parameters. Data are the same as in Figure 1.

The total spectra obtained for the case of the WR-CRs is shown in Figure 2 (bottom panel). We take an injection energy from a single supernova explosion into helium nuclei which is 1.49 times the energy required in the case of the SNR-CRs, and a proton source spectrum cut-off of $1.25 \times 10^{8} \mathrm{GeV}$. The WR-CRs scenario reproduces better both the observed second knee and the position of the ankle.

In Figure 3, we show a comparison of the mean logarithmic mass, $\langle\ln \mathrm{A}\rangle$, obtained using the $X_{\max }$ values measured by different experiments with our model predictions for the Wind-CRs 


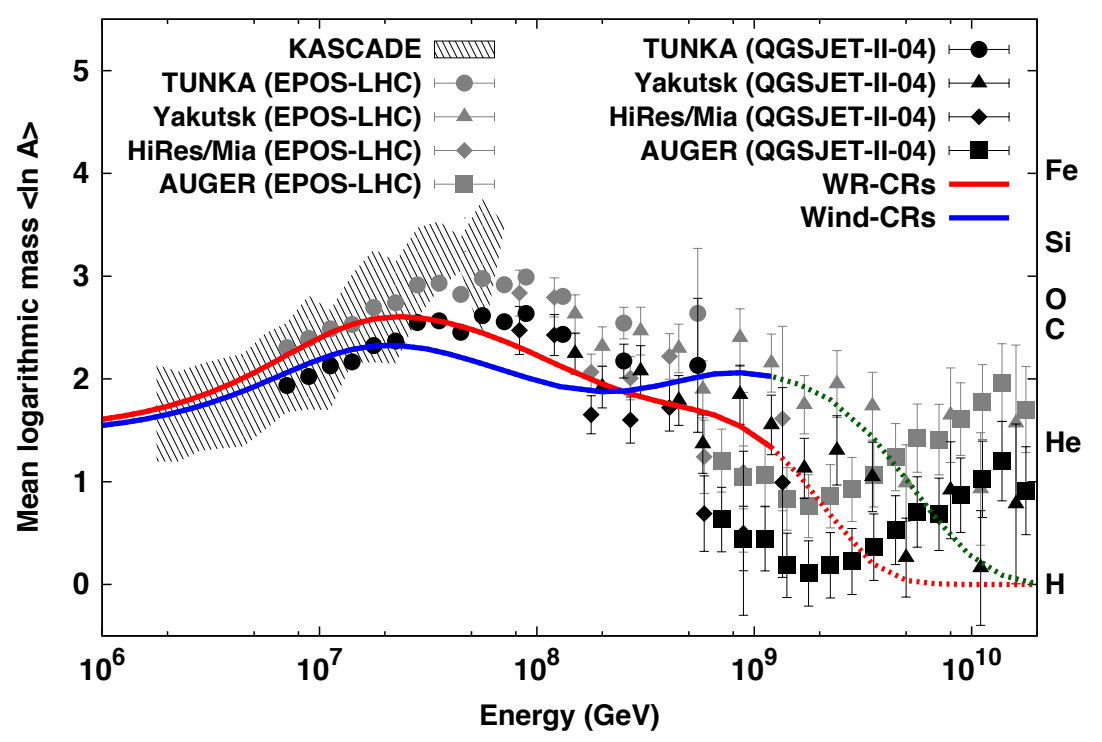

Figure 3: Measured mean logarithmic mass $\langle\log \mathrm{A}\rangle$ of cosmic rays compared to our model predictions. Blue line: Wind-CRs, and Red line: WR-CRs $(\mathrm{C} / \mathrm{He}=0.4)$. Dotted lines represent the region where our predictions may not be fully reliable. Data: KASCADE [11], TUNKA [14], Yakutsk [21], HiRes/Mia [4] and AUGER [1]. The two sets of data corresponds to two different hadronic interaction models (EPOS-LHC and QGSJET-II-04) used in the determination of $\langle\ln \mathrm{A}\rangle$.

and the WR-CRs scenarios. The data consists of two sets which corresponds to two hadronic interaction models used in the determination of $\langle\ln \mathrm{A}\rangle$. Given the large systematic uncertainties of the measurements, both the model predictions seem to agree more or less with the data. However, in the energy range between $\sim 2 \times 10^{7}$ and $2 \times 10^{8} \mathrm{GeV}$, the WR-CRs show a better agreement with the data. Between $\sim 10^{8}$ and $10^{9} \mathrm{GeV}$, the prediction for the Wind-CRs also seem to deviate from the observed trend (changing from heavy to light elements) of the composition. Above $\sim 5 \times 10^{9} \mathrm{GeV}$, the discrepancy between the model predictions and the data is due to the lack of heavy elements in the composition of the extra-galactic cosmic rays considered in our calculation, and is not the focus of our present study.

\section{Conclusion}

We have demonstrated that a single Galactic cosmic-ray component with progressive cut-offs in the energy spectra of different elements cannot explain all the observed features in the all-particle spectrum up to $\sim 10^{9} \mathrm{GeV}$. Using two Galactic components, we have shown that all these observed features as well as the composition can be nicely explained. The first component (SNR-CRs) explains the low-energy measurements and dominates up to $\sim 10^{7} \mathrm{GeV}$, and the second component (Wind-CRs or WR-CRs) dominates beyond this energy up to $\sim 10^{9} \mathrm{GeV}$. In this two-component model, the knee and the second knee are caused by the cut-offs in the energy spectra of the first and the second components respectively. The first component also explains the spectral breaks observed in the proton and helium spectra at $\sim 200 \mathrm{GeV}_{\text {nucleon }}{ }^{-1}$. 


\section{References}

[1] Aab, A., et al. 2014, Phys. Rev. D, 90122006

[2] Aartsen, M. G., et al. 2013, Phys. Rev. D, 88, 042004

[3] Abbasi, R. U., et al. 2009, Astroparticle Physics, 32, 53

[4] Abu-Zayyad, T., et al. 2001, ApJ, 557, 686

[5] Abu-Zayyad, T., et al. 2013, ApJ, 768, L1

[6] Abraham, J., et al. 2010, Physics Letters B, 685, 239

[7] Aharonian, F. A., et al. 2007, A\&A, 464, 235

[8] Adriani, O., et al. 2014, Physics Reports, 544, 323

[9] Aguilar, M., et al. 2014, PRL, 113, 121102

[10] Amenomori, M., et al. 2008, ApJ, 678, 1165

[11] Antoni, T., et al. 2005, Astroparticle Physics, 24, 1

[12] Apel, W. D., et al. 2013, Phys. Rev. D, 87, 081101

[13] Bell, A.R., 2004, MNRAS, 353, 550

[14] Berezhnev, S. F., et al. 2013, in Proc. 33rd ICRC, Paper ID 326

[15] Biermann, P. L., \& Cassinelli, J. P., 1993, A\&A, 277, 691

[16] Bloemen, J. B. G. M., Dogiel, V. A., Dorman, V. L., \& Ptuskin, V. S., 1993, A\&A, 267, 372

[17] Drury, L. O, 1983, Reports on Progress in Physics, 46, 973

[18] Drury, L. O, 2012, Astroparticle Physics, 39, 52

[19] Hillas, A. M., 2006, Journal of Physics: Conference Series, 47, 168

[20] Jokipii, J. R., \& Morfill, G., 1987, 312, 170

[21] Knurenko, S., \& Sabourov, A., 2010, Proc. XVI ISVHECRI

[22] McKee, C. F., \& Ostriker, J. P., 1977, ApJ, 218, 148

[23] Obermeier, A., Ave, M., Boyle, P., et al., 2011, ApJ, 742, 14

[24] Panov, A. D., et al. 2007, Bull. Russ. Acad. Sci., Vol. 71, No. 4, pp. 494

[25] Parizot, E., Marcowith, A., Ballet, J., \& Gallant, Y. A. 2006, A\&A, 453, 387

[26] Pollock, A. M. T., Corcoran, M. F., Stevens, I. R., \& Williams, P. M., 2005, ApJ, 629, 482

[27] Rachen, J. P., Stanev, T., \& Biermann, P. L., 1993, A\&A, 273, 377

[28] Rosslowe, C. K., \& Crowther, P. A., 2015, arXiv:1412.0699

[29] Stanev, T., Biermann, P. L., \& Gaisser, T. K., 1993, A\&A, 274, 902

[30] Thoudam, S., \& Hörandel, J. R. 2013, MNRAS, 435, 2532

[31] Thoudam, S., \& Hörandel, J. R. 2014, A\&A, 567, A33

[32] Thoudam, S., et al., to be submitted to A\&A

[33] Yoon, Y. S., et al. 2011, ApJ, 728, 122

[34] Zirakashvili, V., Breitschwerdt, D., Ptuskin, V. S. \& Völk, H. J., 1996, A\&A, 311, 113

[35] Zirakashvili, V., \& Völk, H. J., 2006, AdSpR, 37, 1923 